\title{
The formation of broad emission line regions in supernova-QSO wind interactions
}

\author{
J. M. Pittard ${ }^{1}$, J. E. Dyson ${ }^{1}$, S. A. E. G. Falle ${ }^{2}$, and T. W. Hartquist ${ }^{1}$ \\ 1 Department of Physics \& Astronomy, The University of Leeds, Woodhouse Lane, Leeds LS2 9JT, UK \\ 2 Department of Applied Mathematics, The University of Leeds, Woodhouse Lane, Leeds LS2 9JT, UK
}

Received 23 March 2001 / Accepted 22 June 2001

\begin{abstract}
We show that a cooled region of shocked supernova ejecta forms in a type II supernova-QSO wind interaction, and has a density, an ionization parameter, and a column density compatible with those inferred for the high ionization component of the broad emission line regions in QSOs. The calculations are based on the assumption that the ejecta flow is described initially by a similarity solution investigated by Chevalier (1982) and Nadyozhin (1985) and is spherically symmetric. Heating and cooling appropriate for gas irradiated by a nearby powerful continuum source is included in our model, together with reasonable assumptions for the properties of the QSO wind. The model results are also in agreement with observational correlations and imply reasonable supernova rates.
\end{abstract}

Key words. hydrodynamics - shock waves - stars: mass-loss - ISM: bubbles - galaxies: active

\section{Introduction}

In active galactic nuclei (AGN), the primary physical mechanism for the excitation of the broad emission line regions (BELR) is photoionization by the underlying broad-band continuum (e.g. Osterbrock \& Matthews 1986; Clavel et al. 1991). Evidence for at least a two-component structure of the BELR has been provided by e.g. CollinSouffrin et al. $(1982,1986)$ and Wills et al.(1985). One component can be identified as consisting of high ionization lines, including Ly $\alpha$, CirI], Civ, HeI, HeII, Nv, and other multiply ionized species, and is known as the HIL. The second component can be identified with the low ionization lines which include the bulk of the Balmer lines, and lines of singly ionized species (e.g. MgII, CII and FeII), and is known as the LIL. The regions emitting the LIL and HIL display different kinematics, as deduced from studies of the profiles and line widths (e.g. Gaskell 1988; Sulentic et al. 1995). In quasars, the HIL are also systematically blue-shifted with respect to the LIL (see various papers in Gaskell et al. 1999). Discussion of the BELR parameters for a range of AGN properties can also be found in the articles in Gaskell et al. (1999).

Many theoretical explanations have been proposed for the origin of the BELR. They include: i) magnetic acceleration of clouds off accretion discs (Emmering et al. 1992); ii) cloud formation in shocks produced by the interaction

Send offprint requests to: J. M. Pittard, e-mail: jmp@ast.leeds.ac.uk of an accretion disc wind with a nuclear wind (Smith \& Raine 1985); iii) the interaction of an outflowing wind with the surface of an accretion disc (Cassidy \& Raine 1996); iv) interaction of stars with accretion discs (Zurek et al.1994); v) tidal disruption of stars in the gravitational field of the BH (Roos 1992); vi) interaction of an AGN wind with supernovae and star clusters (Perry \& Dyson 1985; Williams \& Perry 1994); and vii) emission from accretion shocks (Fromerth \& Melia 2001). Models identified as containing serious difficulties include the formation of BELRs by the thermal instability of a hot optically thin flow (as e.g. discussed by Beltrametti 1981; Shlosman et al. 1985). Perry \& Dyson (1985) noted that this mechanism will not occur when $L_{\mathrm{bol}}>10^{46} \mathrm{erg} \mathrm{s}^{-1}$ as Compton cooling dominates over bremsstrahlung, and therefore it cannot be responsible for the observed BELR in high luminosity QSOs. Two-phase equilibrium models (Krolik et al.1981) are also not applicable to BELRs in these sources because implausibly high values of the AGN mass-loss rate would be required (Perry \& Dyson 1985). The formation of the BELR in ionized red giant or supergiant winds (Scoville \& Norman 1988; Kazanas 1989; Alexander \& Netzer 1994) has difficulty in reproducing the observed broad line wings (Alexander \& Netzer 1997), and models involving the ballistic deceleration of clouds have a number of problems, as summarized by Osterbrock \& Matthews (1986). There are also concerns about the various assumptions in the infalling and orbiting cloud 
models proposed by Kwan and Carroll (Kwan \& Carroll 1982; Carroll 1985; Carroll \& Kwan 1985).

One model which accounts for the LIL emission was proposed by Collin-Souffrin et al. (1988). In this model the LIL emission arises from the surface of the accretion disc, which is illuminated by back-scattered X-rays from the central source. Typical electron densities and absorption columns are inferred to be $n_{\mathrm{e}} \gtrsim 10^{11} \mathrm{~cm}^{-3}$ and $N_{\mathrm{H}} \gtrsim 10^{24} \mathrm{~cm}^{-2}$ respectively. Approximately three quarters of the total luminosity of the broad-line emission is estimated to arise in this fashion (Collin-Souffrin et al.1988). The HIL, therefore, contribute about one quarter of this emission. The characteristic mass of the BELR in high luminosity QSOs is $\sim 100 M_{\odot}$ (Osterbrock 1993), and this is dominated by the HIL.

In this paper we look at one aspect of activity in AGN connected with stars, and which is relevant to the work of Perry \& Dyson (1985). We investigate the interaction of supernova ejecta with the optically thin, low density, hot QSO wind, in the presence of intense continuum radiation. In particular we examine if shocked gas can radiate efficiently enough to cool to temperatures appropriate for the HIL. The evolution of SNRs in a high density static ambient medium has been previously studied by Terlevich et al. (1992), with particular application to the formation of BELRs in starburst models developed to obviate the existence of supermassive black holes in AGNs. Although there are similarities between this work and ours, two differences exist. First, the initial conditions for the supernova ejecta differ from those in our work. Second, these authors did not include Compton cooling or any heating processes in their calculations. These factors will influence the thermal evolution of the shocked regions.

In Sect. 2 we discuss the use of a similarity solution to specify initial conditions in the calculation and the cooling and heating rates adopted. Section 3 contains results for the calculated evolution of a remnant for each of several assumed sets of environmental conditions, showing that the formation of a cool region of shocked ejecta having a density, ionization parameter, and column density in harmony with those inferred from observations occurs for reasonable assumptions. In Sect. 4 we summarize our conclusions and describe how the work can be extended.

\section{Details of the calculations}

Fits to the results of explosion models of type II supernovae indicate that power-law stratifications represent adequate approximations to ejecta density and velocity profiles (e.g. see Woosley et al.1988, and earlier work by Chevalier 1976; Jones et al. 1981), and have been widely used in analytical and numerical studies of remnant evolution. If $\rho \propto r^{-n}$, these results indicate that $n \approx 12$ for the high velocity ejecta.

Self-similar solutions for the structure of the shocked ejecta and swept-up medium assuming power-law approximations for both of the unshocked components are derived by Chevalier (1982) and Nadyozhin (1985), following earlier work by Parker (1963). Their relevance to actual remnants was most recently highlighted by SN 1993J in M 81. High resolution, spatially resolved VLBI observations showed self-similar evolution of the azimuthally averaged radius (Marcaide et al.1997). From application of the Chevalier-Nadyozhin model, the observations are best fitted with $n \approx 12$, in good agreement with results from explosion models.

For $n \geq 3$, there must be an inner core of material with a shallower density profile $\left(\rho \propto r^{-\delta}\right)$ in order for the mass of the ejecta to be finite. Such a core can be seen in the results of explosion models (e.g. Jones et al.1981; Suzuki \& Nomoto 1995). In the simplest case, which we adopt in this paper, a core with uniform density $(\delta=0)$ is surrounded by a steep outer envelope $(n=12)$. The speed of the core radius $v_{\mathrm{c}}$, and the density of the envelope $\rho_{\mathrm{e}}(r)$ are then given by

$$
\begin{aligned}
& v_{\mathrm{c}}=\left[\frac{2(5-\delta)(n-5) E}{(3-\delta)(n-3) M_{\mathrm{ej}}}\right]^{1 / 2}, \\
& \rho_{\mathrm{e}}=g^{n} t^{-3}\left(\frac{r}{t}\right)^{-n}
\end{aligned}
$$

where $E$ is the explosion energy, $M_{\mathrm{ej}}$ is the ejecta mass, and

$g^{n}=\frac{1}{4 \pi(n-\delta)} \frac{[2(5-\delta)(n-5) E]^{(n-3) / 2}}{\left[(3-\delta)(n-3) M_{\mathrm{ej}}\right]^{(n-5) / 2}}$

(cf. Chevalier \& Fransson 1994). For $\delta=0$ and $n=12$ (which we assume in this paper), the density of the core $\rho_{\mathrm{c}}$ is given by

$\rho_{\mathrm{c}}=\frac{729}{1120 \pi}\left(\frac{3}{70} \frac{M_{\mathrm{ej}}^{5}}{E^{3}}\right)^{1 / 2} t^{-3}$

(cf. Band \& Laing 1988), whilst for $\delta=0$ and variable $n$ the relative mass and energy of ejecta in the envelope compared to the core are (cf. Truelove \& McKee 1999):

$$
\begin{aligned}
\frac{M_{\mathrm{c}}}{M_{\mathrm{ej}}} & =\frac{n-3}{n}, \\
\frac{E_{\mathrm{c}}}{E} & =\frac{n-5}{n} .
\end{aligned}
$$

Hence for $n=12,75$ per cent of the mass and 58 per cent of the explosion energy are in the core.

If the explosion occurred in a pure vacuum, the structure of the ejecta would evolve according to Eqs. (1)-(2). However, when the ejecta interact with a surrounding medium, the steep envelope acts as a compressible piston, and the radius of the core relative to the reverse shock increases with time. As long as ejecta in the steep envelope continue to pass through the reverse shock the solution is self-similar, but once the core radius reaches this point the solution abruptly ceases this behaviour.

In this work we adopt the Chevalier-Nadyozhin similarity solutions to specify our initial conditions, with the ejecta distribution being specified by an inner core with $\delta=0$ and an outer envelope with $n=12$. Whilst this is 


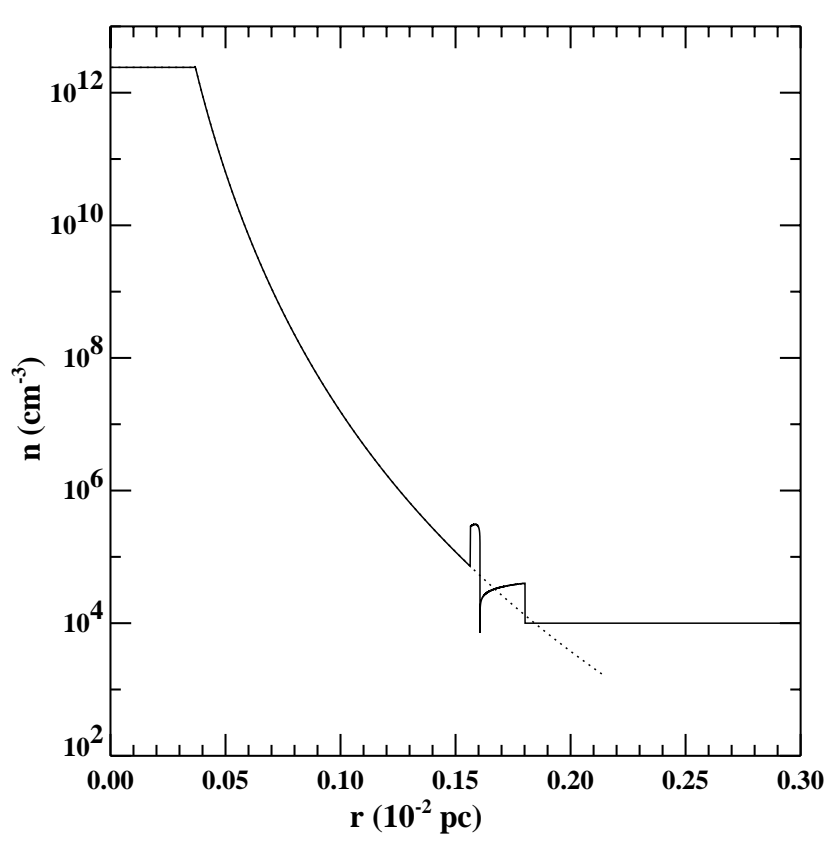

Fig. 1. The density profile for ejecta from a type II SN explosion, modelled as an $n=12$ power-law for the envelope and a $\delta=0$ power-law in the core, an explosion energy of $10^{51} \mathrm{erg}$ and an ejecta mass of $10 M_{\odot}$. The dotted line shows the solution given by Eqs. (1)-(2) which is valid for ejecta expanding into a total vacuum. The solid line shows the case when the progenitor is surrounded by a constant density medium with $n=10^{4} \mathrm{~cm}^{-3}$ : the outer part of the ejecta and the swept-up ambient medium are compressed into the Chevalier-Nadyozhin similarity form.

a simplification to actual distributions obtained from explosion models, at this stage we aim to keep our results as general as possible. It also has the benefit of being easily reproducable and scale-free. In all our calculations we assume a canonical explosion energy of $10^{51}$ ergs and ejecta mass of $10 M_{\odot}$, which is typical of a SN of type II. Figure 1 shows a typical density profile at $t=0.1 \mathrm{yr}$.

Heating and cooling rates for a canonical AGN spectrum were kindly supplied by Tod Woods (cf. Woods et al. 1996) and are included in our calculation. We made use of an adaptive grid hydrodynamic code (see e.g. Falle \& Komissarov 1996, 1998), which is ideally suited to this problem where regions which contain small-scale structure are located within much smoother regions.

To test the accuracy of the code we first imposed the similarity structure on the flow and saw whether it sustained itself. The ambient medium had constant density, zero velocity, and negligible pressure. In Fig. 2 we show the results of this test, which compare favourably with the results from other codes in the recent literature (e.g. Blondin et al. 2000).

The heating and cooling rates are tabulated as functions of temperature and ionization parameter, $\Xi(=F / c p$ where $F$ is the local ionizing flux, $c$ is the speed of light, and $p$ is the gas pressure), and are valid in the optically thin, low-density regime. $\Xi$ is effectively a measure of the ratio of radiation pressure to the gas pressure, and is $2.3 P_{\text {rad }} / P_{\text {gas }}$ for fully ionized gas of cosmic abundance. The heating rates include Compton and photoionization heating of all the ionization stages of hydrogen, helium, and some trace metals (particularly C, N, O, Si, S, and Fe). The cooling includes collisional excitation, recombination cooling, Compton cooling, collisional ionization, and freefree losses. The gas is assumed to be free of dust (Laor \& Draine 1993; but see review by Osterbrock 1993 for an excellent summary of our current understanding of dust in AGN). The rates were calculated using the CLOUDY photoionization code, its standard AGN spectrum, and solar abundances. Whilst abundances in AGN remain a very contentious issue, there is considerable evidence that the nuclear regions are not metal poor, even at high redshift, and also little evidence that the metallicity of the BELR changes with redshift (see Artymowicz et al. 1993). Though it seems certain that nitrogen is overabundant by factors of $\sim 2-9$, particularly for high-redshift sources (e.g. Hamann \& Ferland 1992), most theoretical work has been based on the assumption that the gas is of solar composition: we also apply this assumption. More details of the heating and cooling rates can be found in Woods et al. (1996).

In Fig. 3 we show the thermal equilibrium curve for the assumed AGN spectrum. At low temperatures photoionization heating and cooling due to line excitation and recombination are in near balance. At large temperatures, the equilibrium arises from a balance of Compton heating and cooling. The solutions with positive slope, i.e. $\mathrm{d} T / \mathrm{d} \Xi>0$, are thermally stable (Field 1965). If the slope is negative, solutions can still be thermally stable if the gas cools isochorically, whilst they are thermally unstable if the gas cools isobarically. Note that there is a small range of ionization parameters for which there are stable solutions at intermediate temperatures $\left(T \sim 10^{6} \mathrm{~K}\right)$. The exact shape of this part of the thermal equilibrium curve is poorly known and most likely varies substantially from source to source, since it is a complicated function of the irradiating spectrum, the assumed abundances and thermal processes (cf. Krolik et al. 1981). For a given object it is entirely possible that there are no multi-valued equilibrium temperatures for any $\Xi$. Therefore, in our discussion of the following results we do not assign too much importance to the exact behaviour of the simulations in this part of parameter space since we are not modelling a specific object. In Sect. 3 we further show that the cooling gas often does not pass through this region of parameter space.

To obtain cool gas in thermal equilibrium we require ionization parameters $\Xi \lesssim 10$. As noted by Perry \& Dyson (1985), shocked gas cooled back to equilibrium can have a value of $\Xi$ much lower than its pre-shock value. This is because $\Xi$ does not change if the gas cools isobarically and the post-shock pressure is much greater than the preshock value. Therefore strong shocks can create conditions for the gas to cool to temperatures much lower than the surrounding ambient temperature. 

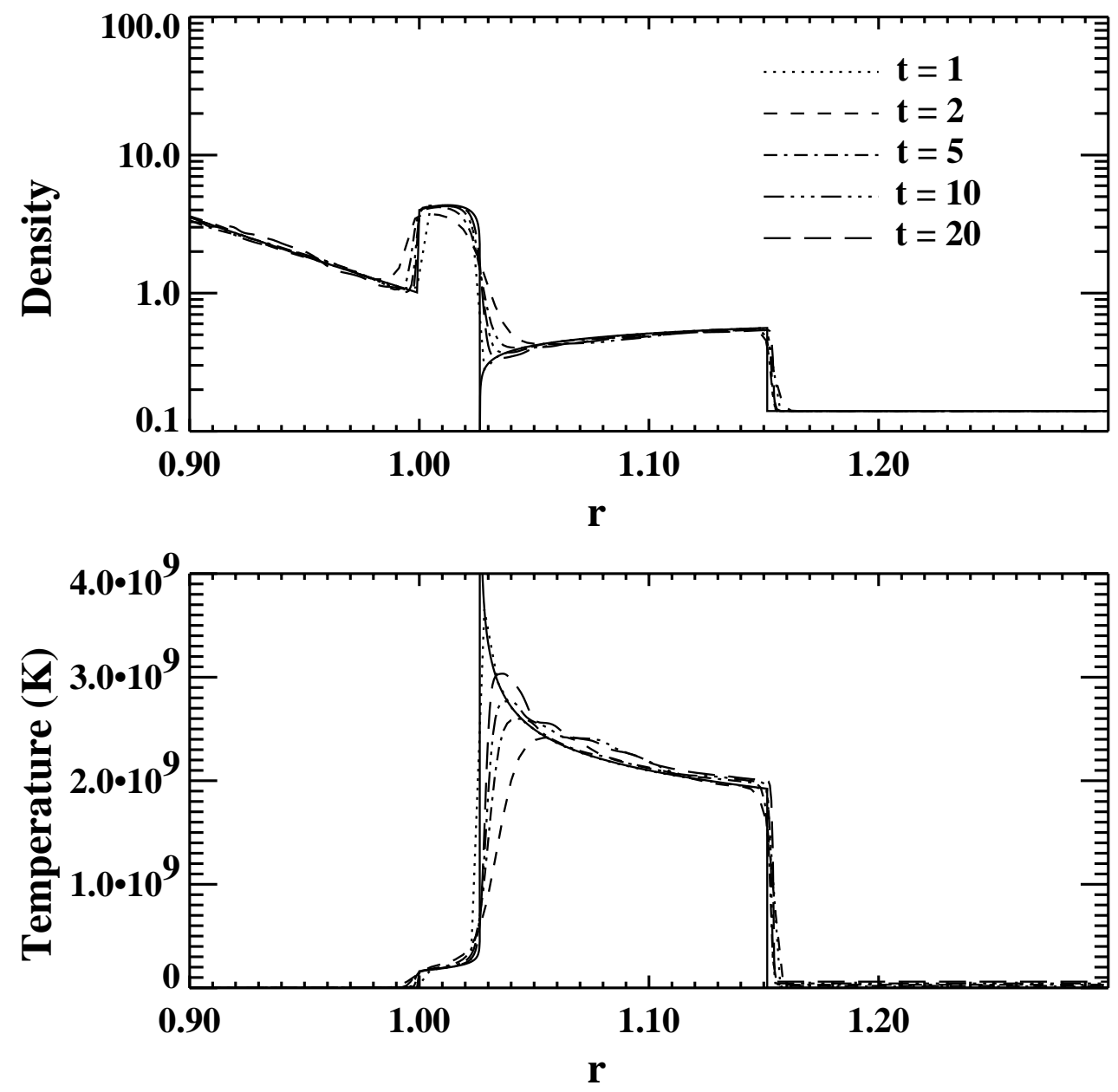

Fig. 2. Comparison of evolved radial profiles of density and temperature rescaled against the similarity solution (solid). The times are measured by the homologous expansion, with $t=1$ corresponding to the initial mapping. The initial number of grid points in the region of shocked ejecta was four. The curves are not coincident because of start-up errors, finite numerical resolution, and unavoidable diffusion and dissipation in the numerical scheme. As the remnant expands the effective resolution of the calculation increases, and the profiles converge towards the original similarity solution.

The ionization parameter of the QSO ISM can be written as

$\Xi_{\mathrm{w}}=2 \times 10^{6} \frac{L_{\mathrm{ion}}}{L_{\mathrm{bol}}} \frac{L_{47}}{n_{\mathrm{w}} r_{\mathrm{pc}}^{2} T_{8}}$,

where $L_{\mathrm{ion}}$ and $L_{\mathrm{bol}}$ are respectively the ionizing and bolometric luminosity of the central source, $L_{47}$ is the bolometric luminosity of the central source in units of $10^{47} \mathrm{erg} \mathrm{s}^{-1}$, $n_{\mathrm{w}}$ is the number density of the wind, $r_{\mathrm{pc}}$ is the distance of the shock from the central source in units of parsecs, and $T_{8}$ is the temperature of the wind in units of $10^{8} \mathrm{~K}$.

The ionization parameter of shocked gas cooled back to equilibrium with the radiation field, $\Xi_{\text {shk }}$, is given by

$\Xi_{\text {shk }}=\frac{T_{8}}{4 \omega^{2}} \Xi_{\mathrm{w}}$,

where $\omega$ is the velocity of the pre-shock gas relative to the shock in units of $0.01 c$ (cf. Perry \& Dyson 1985). For supernovae in the central regions of QSO, this velocity is a combination of the velocity of the blast wave, the velocity of the ambient medium, and the Keplerian velocity of the progenitor star. The QSO medium has characteristic velocities of $v_{\text {wind }} \sim 1000-3000 \mathrm{~km} \mathrm{~s}^{-1}$ ( $\omega=0.3-1.0$, Williams et al.1999), whilst the expansion velocities of young ejecta-dominated SNRs satisfying the Chevalier-Nadyozhin similarity solution can be greater than $10000 \mathrm{~km} \mathrm{~s}^{-1}$. Therefore, it is not difficult to obtain $\Xi_{\text {shk }} \lesssim 1$ for which $T_{\text {eq }} \sim 10^{4} \mathrm{~K}$ (see Fig. 3). The crucial question is whether the shocked gas remains at high pressures long enough to cool from its post-shock temperature to $T \sim 10^{4} \mathrm{~K}$. This requires that the dynamical timescale for the SNR is longer than the cooling timescale of the post-shock gas.

\section{Results}

\subsection{Adiabatic evolution}

We initially investigate the adiabatic evolution of a SNR surrounded by a static, constant density medium with $n_{\mathrm{w}}=10^{6} \mathrm{~cm}^{-3}$ and $T_{\mathrm{w}}=1.33 \times 10^{7} \mathrm{~K}$. Whilst this value of $n_{\mathrm{w}}$ is marginally optically thick to X-rays (at the Fe K-shell edge) and to Compton scattering for distances 


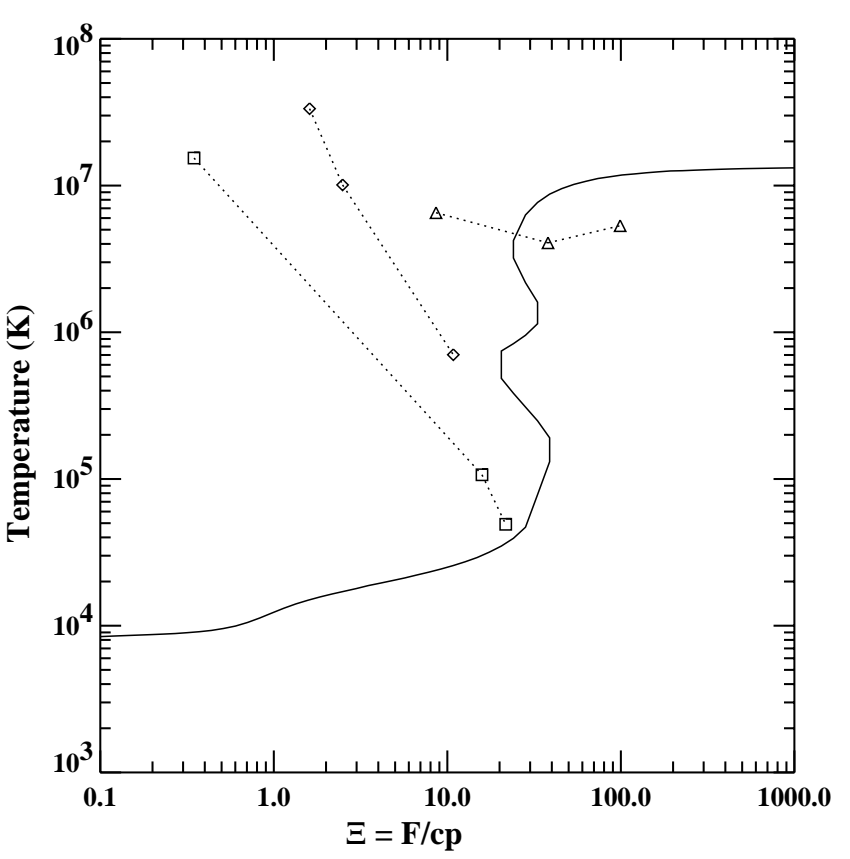

Fig. 3. Thermal equilibrium curve for the standard AGN spectrum in CLOUDY (see Woods et al. 1996). The symbols refer to the thermal evolution of the cool region in Models A (diamonds), B (triangles) and C (squares) (Figs. 5-7 respectively).

greater than $\approx 0.5 \mathrm{pc}$, it is of no concern provided that the density falls with radius on similar scales. However, as our results show, the radius of the supernova remnant remains significantly smaller than this scale. We note also that our chosen ambient density is similar to that found by Williams et al. (1999). In the central regions of AGN, the ISM density is primarily determined by mass-loss from the central stellar cluster through winds and supernova explosions. Collisions and tidal disruptions are negligible except in the central regions of the densest clusters. Compared to the central cluster, the mass flux from the external ISM (e.g. flow into the nucleus from a galactic bar) is unlikely to provide a significant fraction of the fuelling requirements of the AGN as a continuous source (Shlosman et al.1990). Our value of $T_{\mathrm{w}}$ is also characteristic of the Compton temperature in a hard QSO radiation field.

The assumption of a static ambient medium keeps the problem in its simplest form. For the unshocked ejecta we set $T=10^{4} \mathrm{~K}$. The Chevalier-Nadyozhin similarity solution is derived on the assumption of negligible thermal energy in the unshocked ejecta and ambient medium, and our specified temperature implies thermal pressures orders of magnitude below the relevant ram pressure. Whilst this also implies exceedingly high absorption columns through the ejecta core, the covering fraction is very small.

In Fig. 4 the radius and speed of the contact discontinuity are shown as functions of time. As discussed earlier, the solution remains self-similar until the reverse shock propagates to the edge of the core: this occurs at $t \approx 9 \mathrm{yr}$, and is reflected in the structure of the curves in Fig. 4.

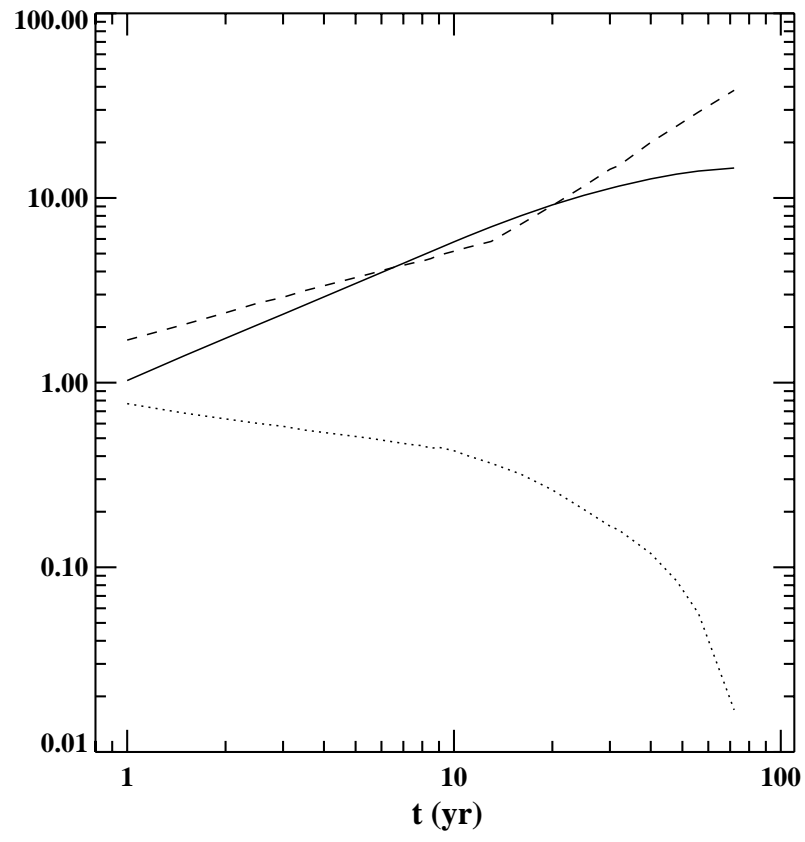

Fig. 4. Evolution of the radius (solid) and speed (dots) of the contact discontinuity of an adiabatic SNR as a function of time (arbitrary units). At $t=1 \mathrm{yr}$, their respective values are 1.0264 and 0.7698. The SNR evolves in a self-similar fashion with $r \propto t^{3 / 4}$ and $v \propto t^{-1 / 4}$ until the reverse shock reaches the radius of the ejecta core. This occurs at $t \approx 9 \mathrm{yr}$. Also shown is the time evolution of $\Xi$ (dashes), which at $t=1 \mathrm{yr}$ has a value of 1.7. Prior to $t \approx 9 \mathrm{yr}$ it evolves as $t^{1 / 2}$, but increases in value much more rapidly for $t \gtrsim 13 \mathrm{yr}$.

Although we have not included heating and cooling terms in this calculation, it is instructive to assume a radiation field and to plot the evolution of the ionization parameter of the equilibrium temperature of the shocked gas. This gives an impression of whether the shocked gas is likely to heat or cool if these terms are included. Assuming that the remnant is $0.33 \mathrm{pc}$ distant from a central ionizing source of $L_{\text {ion }}=10^{47} \mathrm{erg} \mathrm{s}^{-1}$, we show in Fig. 4 the time evolution of this parameter in the shocked ambient medium. This position was chosen because, with the assumption of constant ionizing flux, $\Xi$ tracks the inverse of $p$ and the profile of $p$ is flatter at this point. Figure 4 shows that $\Xi$ initially evolves as $t^{1 / 2}$, but increases more rapidly once the flow diverges from self-similarity. This is due to a large increase in the volume of the shocked region (as the reverse shock begins to move back towards the centre of the remnant in the Lagrangian frame) at roughly constant total energy, which leads to a reduction in the internal energy per unit volume and hence pressure, and the corresponding increase in $\Xi$. It is therefore apparent that the formation of cool clouds in a SNR shock is favoured at early times, when the pressure of the shocked region is high and $\Xi$ is low. In particular, since $\Xi$ rapidly increases once the ejecta core reaches the reverse shock, a cool region has the best opportunity to form before this point. Therefore we equate the dynamical timescale of the remnant, $t_{\mathrm{dyn}}$, with the time at which the ejecta core reaches 
the reverse shock, and require that the shocked gas has a cooling time, $t_{\text {cool }}$, shorter than this. We derive expressions for $t_{\mathrm{dyn}}$ and $t_{\text {cool }}$ in Appendix A.

We also require that the ionization parameter $\Xi$ of the post-shock gas at $t=t_{\mathrm{dyn}}$ is low enough to allow the gas to cool to $T \approx 2 \times 10^{4} \mathrm{~K}$. Cold gas will not form if $\Xi \gtrsim \Xi_{\text {crit }}$ (where $\Xi_{\text {crit }} \lesssim 30$ for the AGN spectrum used to generate the thermal equilibrium curve in Fig. 3) whether or not $t_{\text {cool }} \lesssim t_{\text {dyn }}$. We derive an expression for this condition also in Appendix A. Hence for cold gas to form we need to satisfy $t_{\text {cool }} \lesssim t_{\text {dyn }}$ and $\left.\Xi\right)_{t_{\text {dyn }}} \lesssim \Xi_{\text {crit }}$. Both of these conditions are evaluated for each of the following models.

\subsection{Radiative evolution}

We now include the full heating and cooling rates in all of our remaining simulations. We start the evolution on the hydrodynamic grid at a time early enough for the dominant cooling of the shocked gas to be adiabatic expansion. As the remnant expands, radiative cooling gradually increases in importance, and a smooth transition from the adiabatic self-similar solution into the radiative regime occurs. Because the post-shock temperatures can be very high early on $\left(T \gtrsim 10^{9} \mathrm{~K}\right)$, we extrapolate the heating and cooling functions used by Woods et al. (1996) with a second order polynomial. This should be a reasonable approximation to the true rates because over the range $10^{8} \lesssim T \lesssim 10^{9} \mathrm{~K}$, cooling and heating are dominated by Compton or bremmstrahlung processes which are smoothly varying functions of $T$. Furthermore, since the cooling of the remnant is initially dominated by adiabatic expansion, the inaccuracies in the correct rates from this extrapolation are small. Finally, as the remnant expands, the pre-shock ejecta are slowly heated from $T=10^{4} \mathrm{~K}$ to $\sim 10^{6} \mathrm{~K}$. We assume again that the remnant is $0.33 \mathrm{pc}$ distant from a central source with $L_{\text {ion }}=10^{47} \mathrm{erg} \mathrm{s}^{-1}$. The parameters for this model (Model A) are listed in Table 1.

In Fig. 5 the evolution of the shocked region is shown. We find that the shocked ejecta substantially cool with heating and cooling rates included in the calculation. By $t=1.0 \mathrm{yr}$ a cool region has formed with a temperature less than that of the undisturbed ambient medium. At $t=1.4 \mathrm{yr}$ it has a temperature of $7.0 \times 10^{5} \mathrm{~K}$. Divergence from the adiabatic self-similar solution can be seen in the time evolution of the $T, \Xi$, and $\rho$ profiles. In the temperature profiles, this is first manifested as a change in the slope of the region of shocked ambient material from $\mathrm{d} T / \mathrm{d} r>0$ to $\mathrm{d} T / \mathrm{d} r<0$. The formation of the cool region at later times is of course a much larger divergence from the self-similar solution. In the density profiles, the formation of the cool region is apparent as a sharp coincident growth in density. The profiles of ionization parameter show the gradual temporal increase in $\Xi$ expected from the evolution of the self-similar solution. However, at $t=1.4 \mathrm{yr}$, the value of $\Xi$ associated with the cool material is substantially larger than the value of the immediate surroundings. This is due to the radiative losses
Table 1. Parameters for the models considered in this paper. All models have the same ionization parameter and temperature for the ambient medium $\left(\Xi \approx 150, T_{\mathrm{w}}=1.33 \times 10^{7} \mathrm{~K}\right)$.

\begin{tabular}{lllll}
\hline Model & $\begin{array}{l}n_{\mathrm{w}} \\
\left(\mathrm{cm}^{-3}\right)\end{array}$ & $\begin{array}{l}v_{\mathrm{w}} \\
\left(\mathrm{km} \mathrm{s}^{-1}\right)\end{array}$ & $\begin{array}{l}F_{\text {ion }} \\
\left(\mathrm{erg} \mathrm{cm}^{-2} \mathrm{~s}^{-1}\right)\end{array}$ & $\begin{array}{l}\text { Cool } \\
\text { Regions }\end{array}$ \\
\hline $\mathrm{A}$ & $10^{6}$ & 0 & $7.67 \times 10^{9}$ & $\mathrm{Y}$ \\
$\mathrm{B}$ & $10^{4}$ & 0 & $7.67 \times 10^{7}$ & $\mathrm{~N}$ \\
$\mathrm{C}$ & $10^{4}$ & 3000 & $7.67 \times 10^{7}$ & $\mathrm{Y}$ \\
\hline
\end{tabular}

becoming so high that cooling no longer takes place isobarically. In the limit that the cooling timescale, $t_{c}$, is much less than the appropriate dynamical timescale, $t_{\mathrm{d}}$, the cold gas would cool isochorically. Here $t_{\mathrm{d}}$ is the timescale for the hot post-shock gas to respond to the rapid depressurization of this material, and is of the order of the timescale for collapse of the reverse shock, $\approx l /\left(v_{\mathrm{cd}}-v_{\mathrm{rs}}\right)$, where $l$ is the length scale of the shocked ejecta, and $v_{\mathrm{cd}}\left(v_{\mathrm{rs}}\right)$ is the velocity of the contact discontinuity (reverse shock). The thermal parameters of the cool region at $t=0.5,1.0$, and $1.4 \mathrm{yr}$ are plotted as crosses in Fig. 3.

Beyond $t=1.4 \mathrm{yr}$, as the cloud cools further, its thermal energy as a fraction of its total energy (thermal plus kinetic) approaches the round-off error of our calculation $\left(\sim 10^{-4}\right)$, and we cannot follow its evolution past this time. There is also the well-known problem of the "eating away" of the edges of hot material. This affects all numerical schemes, and results from the numerics smearing the temperature gradient and placing cells at intermediate temperatures, which then undergo high cooling (often the linecooling bump at $T \sim 10^{5} \mathrm{~K}$ ). In this fashion cool regions can rapidly grow as hot regions are "eaten away".

However, it is clear that the gas will continue to cool, and eventually reach $T \approx 2 \times 10^{4} \mathrm{~K}$, as the following argument demonstrates. With $t_{\mathrm{c}}<<t_{\mathrm{d}}$ the gas cools isochorically so $p \propto T \propto 1 / \Xi$. At $t=1.4 \mathrm{yr}$, the cloud is at a temperature $T=7.0 \times 10^{5} \mathrm{~K}$ and an ionization parameter $\Xi=10.8$. It therefore moves approximately along a line of slope -1 in Fig. 3, and encounters the thermal equilibrium curve at $\Xi \approx 40$ and $T \approx 2 \times 10^{5} \mathrm{~K}$. This point is thermally stable and the rapid decrease in the temperature is brought to a halt. The temperature of the cool region, $T_{\mathrm{c}}$, then remains relatively constant, and ajusts only for changes in the value of the ionization parameter, $\Xi_{\mathrm{c}}$, which occur on a sound-crossing timescale as the surrounding post-shock material gradually repressurizes the cool gas to its level. The sound crossing time within the shocked ejecta is short compared to the dynamical time of the remnant, so $\Xi_{\mathrm{c}} \rightarrow \Xi_{\mathrm{ss}}$, where $\Xi_{\mathrm{ss}}$ is the ionization parameter of the surrounding shock material $\left(\Xi_{\mathrm{ss}} \approx 2-3\right.$ in the shocked ambient gas). There is therefore no immediate danger of $\Xi_{\mathrm{c}}$ increasing to the point that the cooled gas begins to reheat towards the Compton temperature. Thus $\Xi_{\mathrm{c}}$ approaches $\Xi_{\mathrm{ss}}$ and the cooled region moves along 

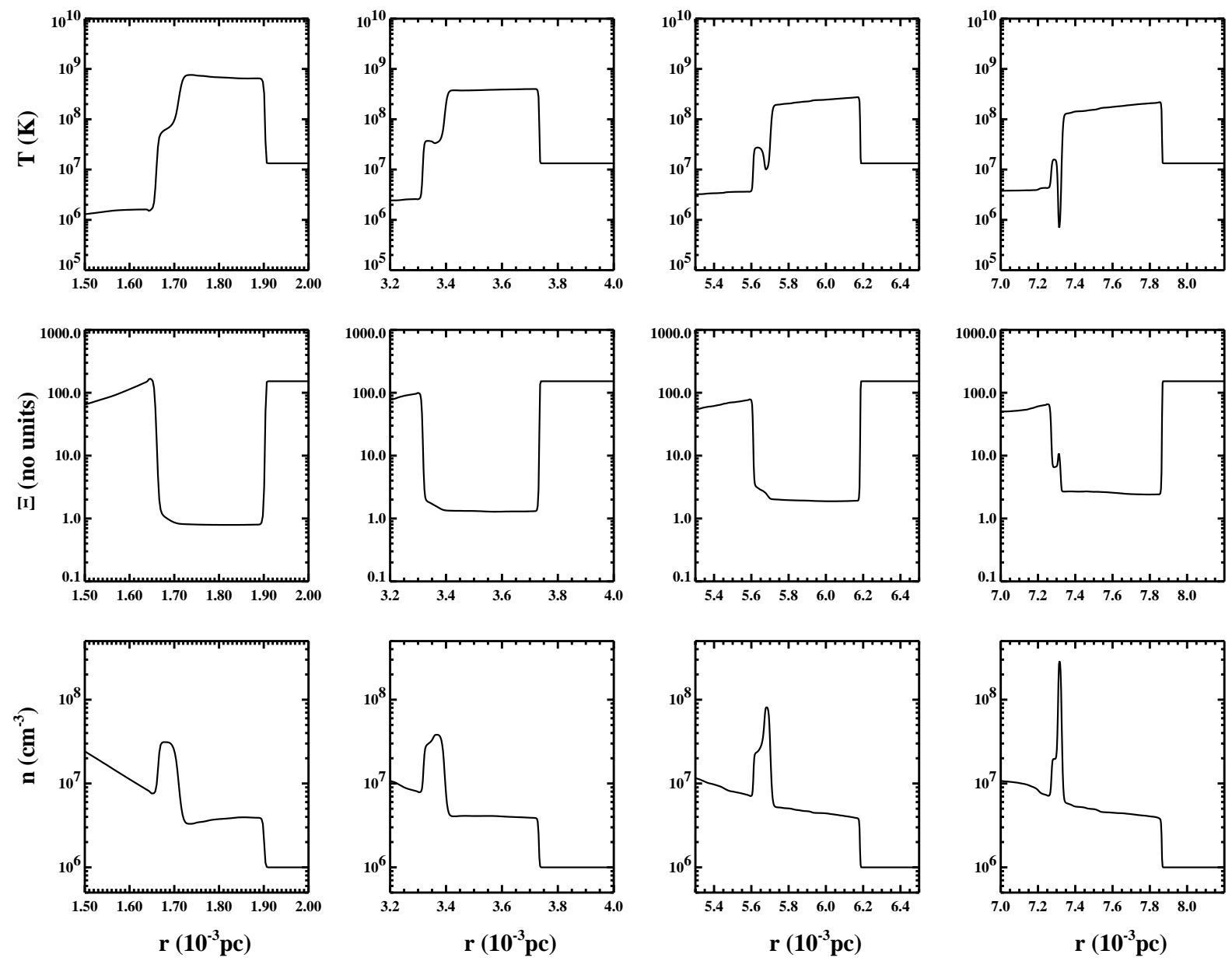

Fig. 5. The evolution of the remnant of a type-II supernova for a constant density medium with $n_{\mathrm{w}}=10^{6} \mathrm{~cm}^{-3}$ and $\Xi_{\mathrm{w}} \approx 150$ (Model A). The medium is stationary with respect to the remnant, and the entire area is immersed in a radiation field appropriate to an AGN of ionizing luminosity $10^{47} \mathrm{erg} \mathrm{s}^{-1}$ with the central engine at a distance of $0.33 \mathrm{pc}$. The panels show from top to bottom the evolution of the temperature, ionization parameter, and density of the region of hot shocked gas. This is bounded on its right edge by the forward shock propagating into the ambient medium and on its left edge by the reverse shock. From left to right the corresponding times are $0.2 \mathrm{yr}, 0.5 \mathrm{yr}, 1.0 \mathrm{yr}$ and $1.4 \mathrm{yr}$. The formation of a cooled region of gas can clearly be seen.

the thermal equilibrium curve to the left. The density of the region, $n_{\mathrm{c}}$, increases during this process until

$n_{\mathrm{c}}=\frac{T_{\mathrm{c}^{\prime}}}{T_{\mathrm{c}}} n_{\mathrm{c}^{\prime}}$,

where $n_{\mathrm{c}^{\prime}}\left(T_{\mathrm{c}^{\prime}}\right)$ is the density (temperature) of the cooled material at the end of our simulation. From the results in Fig. 5, we obtain $n_{\mathrm{c}} \approx$ few $\times 10^{10} \mathrm{~cm}^{-3}$, in good agreement with inferred values of the density of the HIL region from observations. We can also estimate the resultant hydrogen column of the region: at $t=1.4 \mathrm{yr}$, its thickness is $\approx 2 \times 10^{13} \mathrm{~cm}$ (the numerical resolution of our calculation is about half this), and its density is $\approx 3 \times 10^{8} \mathrm{~cm}^{-3}$, which for solar abundances results in a column density of $\approx 10^{22} \mathrm{~cm}^{-2}$, again in agreement with observations of the HILs. The radius of the remnant is $\approx 7.5 \times 10^{-3} \mathrm{pc}$ at this stage, whilst the expansion speed of the contact discontinuity (and thus the velocity of the cool region) is $\approx 4 \times 10^{8} \mathrm{~cm} \mathrm{~s}^{-1}$.
Observations of the HILs have revealed that the optical depth is less than but of order unity, and that $N_{\mathrm{H}} \approx 10^{22} \mathrm{~cm}^{-2}$. The observed range of the ionization parameter of the HIL region is $0.3 \lesssim \Xi \lesssim 2$ (Kwan \& Krolick 1981), which is somewhat higher than that inferred for the LIL region. The systematic blue-shift of the HILs with respect to the LILs is interpreted as the HILs forming in a region with bulk outflow (possibly inflow) and that the red-shifted emission is obscured. Line widths, which reflect local gas velocities, are typically several $10^{3} \mathrm{~km} \mathrm{~s}^{-1}$. Hence our model results are in harmony with the temperature, ionization parameter, column density, and velocity dispersion of the observed HIL BELR clouds.

We now check to see if our results are in agreement with the two conditions which must be satisfied for the shocked gas to cool down to $T \approx 2 \times 10^{4} \mathrm{~K}$, namely $t_{\text {cool }} \lesssim t_{\text {dyn }}$ and $\left.\Xi\right)_{t_{\mathrm{dyn}}} \lesssim \Xi_{\text {crit }}$. For $n=12, \delta=0$, $\mathrm{E}=10^{51} \operatorname{ergs}, M_{\mathrm{ej}}=10 M_{\odot}, R_{2} / R_{\mathrm{c}}=0.974, \mathrm{~A}=0.19$ and $\rho_{\mathrm{w}}=10^{-18} \mathrm{~g} \mathrm{~cm}^{-3}$, we obtain from Eq. (A.5) 

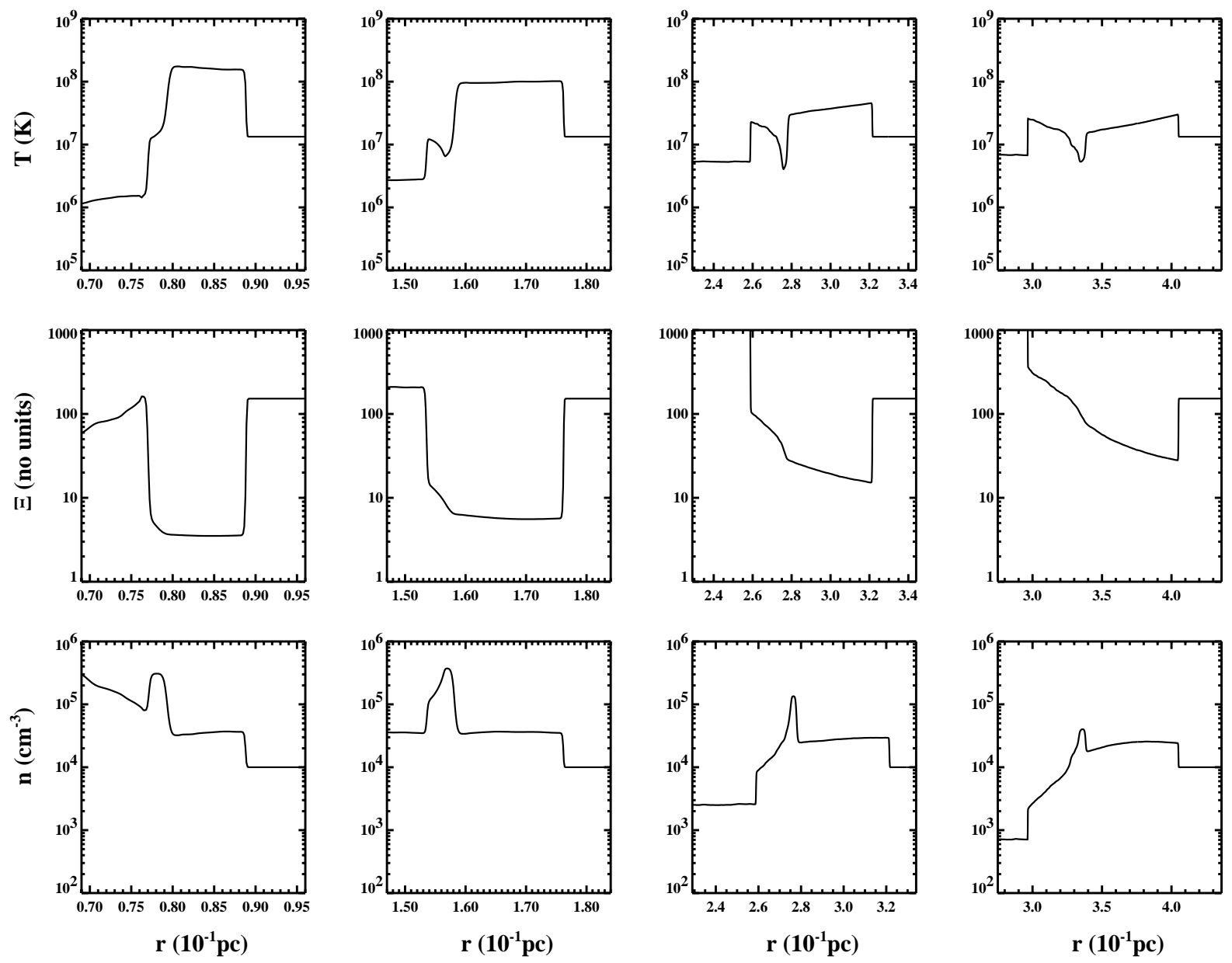

Fig. 6. As Fig. 5 (Model A) but for a constant density medium with $n_{\mathrm{w}}=10^{4} \mathrm{~cm}^{-3}$, an ionizing AGN luminosity of $10^{46}$ erg s${ }^{-1}$, and a distance of $1 \mathrm{pc}$ to the central engine (Model B). $\Xi_{\mathrm{w}}$ is again $\approx 150$. From left to right the corresponding times are $20 \mathrm{yr}$, $50 \mathrm{yr}, 120 \mathrm{yr}$ and $180 \mathrm{yr}$. In contrast to Model A, cool clouds have no opportunity to form since the interaction of the ejecta core with the reverse shock leads to a rapid depressurization of the shocked gas.

$t_{\mathrm{dyn}}=7 \mathrm{yr}$. Assuming solar abundance for the ejecta, $\mu_{\mathrm{s}}=0.61$, and $t_{\text {cool }}=3 \mathrm{yr}$. Hence $t_{\text {cool }}<t_{\text {dyn }}$ as required. The fact that the cooled region first forms at $t \approx 1.4 \mathrm{yr}$ indicates that we are underestimating the cooling rate at lower temperatures where line-cooling is dominant. For these parameters, we further obtain from Eq. (1) $v_{\mathrm{c}}=3610 \mathrm{~km} \mathrm{~s}^{-1}$, and from Eq. (A.13) $\left.\Xi_{\mathrm{shk}}\right)_{t_{\mathrm{dyn}}}=6.3$, and thus Model A also satisfies the requirement that $\left.\Xi_{\text {shk }}\right)_{t_{\text {dyn }}} \lesssim 20$. In reality the ejecta will be enriched in metals, which will reduce the above estimate of $t_{\mathrm{cool}}$.

\subsection{Exploration of associated parameter space}

\subsubsection{Variation of $n_{\mathrm{w}}, r_{\mathrm{pc}}$, and $L_{\mathrm{ion}}$}

Since $n_{\mathrm{w}}, r_{\mathrm{pc}}$, and $L_{\mathrm{ion}}$ all influence $\Xi_{\mathrm{shk}}$ it is impossible to discuss the effect of a variation in one without also considering the impact of the others. This inevitably leads to some complexities but we attempt to keep our discussion as simple as possible.

We can first consider the effect of varying the assumed ambient density. Since the equilibrium temperature is a function of $\Xi$, we wish to keep this value constant for our initial investigation. As $\Xi_{\mathrm{w}} \propto L_{\mathrm{ion}} /\left(n_{\mathrm{w}} r_{\mathrm{pc}}^{2}\right)$, this implies a variation in flux from the central engine, which can obviously be interpreted as a variation in the ionizing luminosity $L_{\text {ion }}$ with $r_{\mathrm{pc}}$ fixed, or as a variation in the distance $r_{\mathrm{pc}}$ between the remnant and the central engine with $L_{\text {ion }}$ fixed, or a suitable variation in both $L_{\text {ion }}$ and $r_{\mathrm{pc}}$.

In Fig. 6 we show the results for a simulation with $\Xi_{\mathrm{w}} \sim 150$ (matching the earlier simulation of Model A shown in Fig. 5) but with a lower ambient density $\left(n_{\mathrm{w}}=10^{4} \mathrm{~cm}^{-3}\right)$ and AGN flux (e.g. $L_{\text {ion }}=10^{46} \mathrm{erg} \mathrm{s}^{-1}$ with the remnant 1 pc distant). We call this Model B. As a result of the lower ambient density, it has a longer evolution time $\left(t_{\mathrm{dyn}}=32 \mathrm{yrs}\right)$ and the remnant expands further than in Model A. Relative to Model A, the lower flux from the central engine also increases the cooling timescale of the shocked gas $\left(t_{\mathrm{cool}}=300 \mathrm{yrs}\right)$. Although the ionziation parameter of the shocked gas at $t=t_{\mathrm{dyn}}$ is less than $\Xi_{\text {crit }}$ $\left[\Xi_{\mathrm{shk}}\right)_{t_{\mathrm{dyn}}}=6.4$, as for Model A], because $t_{\text {cool }}>t_{\mathrm{dyn}}$ we do not expect any of the shocked material to cool to $T \approx 2 \times 10^{4} \mathrm{~K}$.

Once the reverse shock reaches the core radius at $t=32 \mathrm{yr}$, the solution swiftly diverges from a self-similar form (despite the cooling of the post-shock material, many 
aspects such as the radius and velocity of the shocks are not too far removed from such an evolutionary form prior to this point). At this time the coolest shocked gas is at $T \approx 7 \times 10^{6} \mathrm{~K}$. The subsequent acceleration of the reverse shock towards the centre of the remnant rapidly de-pressurizes the shocked region, increasing the ionization parameter of the shocked gas, and ultimately leading to its reheating towards the Compton temperature. Thus, by simply adjusting the density of the ambient medium, whilst keeping all other parameters constant, we have shown that a cool region may not form if the effective ram pressure of the ambient medium is not sufficiently high.

We consider whether there is also a mechanism which prevents the formation of cool regions in SNRs once the density of the ambient material increases past some limit. This question can be addressed in the following way. As $n_{\mathrm{w}}$ increases, $L_{\mathrm{ion}} / r_{\mathrm{pc}}^{2}$ must similarly increase to maintain a given value for $\Xi_{\mathrm{w}}$. For a given $L_{\text {ion }}$ this means a reduction in $r_{\mathrm{pc}}^{2}$ (which might be consistent with an increase in $n_{\mathrm{w}}$ ). A higher value of $n_{\mathrm{w}}$ leads to a faster evolution of the remnant, which given that the cooling timescale of the shocked gas also shortens, does not prevent the possibility of cool regions forming. However, physically it does mean that the lifetime of the cool regions is also very short, since the evolved time between their formation and their destruction, which presumably occurs shortly after the interaction of the ejecta core with the reverse shock, is short too. Hence at some value of $n_{\mathrm{w}}$, the contribution to the BELR emission will be on such short timescales that it will become an unimportant component of the overall emission.

We note that it is unlikely that the ejecta density actually has such a sharp cut in its gradient. However, once the ejecta passing through the reverse shock starts deviating from an $r^{-12}$ profile the volume of the shocked region will start expanding with an inevitable rise in the ionization parameter. This would occur at an earlier time relative to the sharp cut-off case and would tend to reduce the contribution of mass to the BELR by this model. Assuming a constant value of $\Xi_{\mathrm{w}}$ and $L_{\text {ion }}$, this argument also introduces a lower bound to the value of $r_{\mathrm{pc}}$ at which a cool region can form.

Conversely, for a given $r_{\mathrm{pc}}$, an increase in $n_{\mathrm{w}}$ and constant $\Xi_{\mathrm{w}}$ means a corresponding increase in $L_{\text {ion }}$. In a similar way to the above argument, this introduces an upper bound to the value of $L_{\text {ion }}$ at which a cool region can form. These conditions together place upper bounds on the values of $n_{\mathrm{c}}$ and $v_{\mathrm{c}}$ inferred from observations.

\subsubsection{Variation of $\Xi_{\mathrm{w}}$}

We now consider the effect of a different wind ionization parameter on the possible formation of a cool BELR. In our simulations so far we have deliberately specified a value of $\Xi_{\mathrm{w}}$ which is high enough for the ambient medium to exist at the Compton temperature, but which is at the same time almost as low as we could make it, providing us with the best opportunity to form a cool region (through a low value of $\Xi_{\text {shk }}$ cf. Eq. (8)). If $\Xi_{\mathrm{w}}$ was increased in value (and given that $T_{\mathrm{w}}$ is almost constant for large values of $\Xi_{\mathrm{w}}$ ) we would require a higher relative velocity, $\omega$, to obtain a given value of $\Xi_{\text {shk }}$ (cf. Eq. (8)). For a given remnant age and $n_{\mathrm{w}}$, and assuming a static medium, a higher value of $\Xi_{\mathrm{w}}$ gives a higher value of $\Xi_{\mathrm{shk}}$, and since $\Xi_{\text {shk }}$ gradually increases with time as the expansion velocity of the remnant slows, this effectively reduces the "window" of opportunity where $\Xi_{\text {shk }}$ is low enough for $T_{\text {eq }} \sim 10^{4} \mathrm{~K}$. This in turn places a limit on the maximum cooling timescale for the shocked gas, which for a given value of $\Xi_{\mathrm{w}}$, feeds back into a lower limit on the ambient density, $n_{\mathrm{w}, \min }$. If $n_{\mathrm{w}}<n_{\mathrm{w}, \min }$, the shocked gas will not have enough time to cool to $\sim 10^{4} \mathrm{~K}$ before $\Xi_{\text {shk }}$ becomes too large.

\subsubsection{Variation of $\omega$}

If the medium surrounding the remant has some bulk velocity of its own, it is possible to obtain the same value of $\Xi_{\text {shk }}$ at the same remnant age, for a higher value of $\Xi_{\mathrm{w}}$. On the side of the remnant facing the oncoming "AGN wind" the expansion of the remnant is inhibited, leading to an increased value for $v_{\text {r }}$ (cf. Eqs. (A.8) and (A.9)) or alternatively $\omega$ (cf. Eq. (8)). In turn this reduces the critical density of the ambient medium needed for a cool region to form, $n_{\mathrm{w}, \min }$. In Fig. 7 we show the results for Model C, a remnant of a type-II supernova expanding into a constant density medium with $n_{\mathrm{w}}=10^{4} \mathrm{~cm}^{-3}, \Xi_{\mathrm{w}} \approx 150$, and a flow velocity of $3000 \mathrm{~km} \mathrm{~s}^{-1}$ (cf. Williams et al. 1999) towards the remnant. These results are indicative of the remnant structure on the upstream side (since the shocked region is thin with respect to the radius of the remnant). The entire area is again immersed in a radiation field appropriate to a QSO of luminosity $10^{46} \mathrm{erg} \mathrm{s}^{-1}$ with the central engine at a distance of 1 pc. Figure 7 shows that this time a cool region can form. In this case the ionization parameter of the cool gas is expected to be $\approx 0.5$ and its density to be $n \approx 10^{9} \mathrm{~cm}^{-3}$. Its column density is $\approx 6 \times 10^{21} \mathrm{~cm}^{-2}$ and its speed is $\approx 2580 \mathrm{~km} \mathrm{~s}^{-1}$. These values are again in good agreement with observations.

\subsubsection{Constraints on the parameter range}

The previous sections show that there are two broad constraints on the parameter range required for cool regions to form in SNRs. First, the post-shock ionization parameter needs to be low enough for the equilibrium temperature of this region to approach $\approx 10^{4} \mathrm{~K}$. This requires that the SNR evolves in a dense medium (Model A (Fig. 5) vs. Model B (Fig. 6)) but at the same time is not too close to the central engine (cf. Eq. (A.13)). Whilst tending to decrease the evolutionary timescale of the remnant, a higher effective confining pressure increases the density of the shocked gas leading to shorter cooling timescales. Thus 

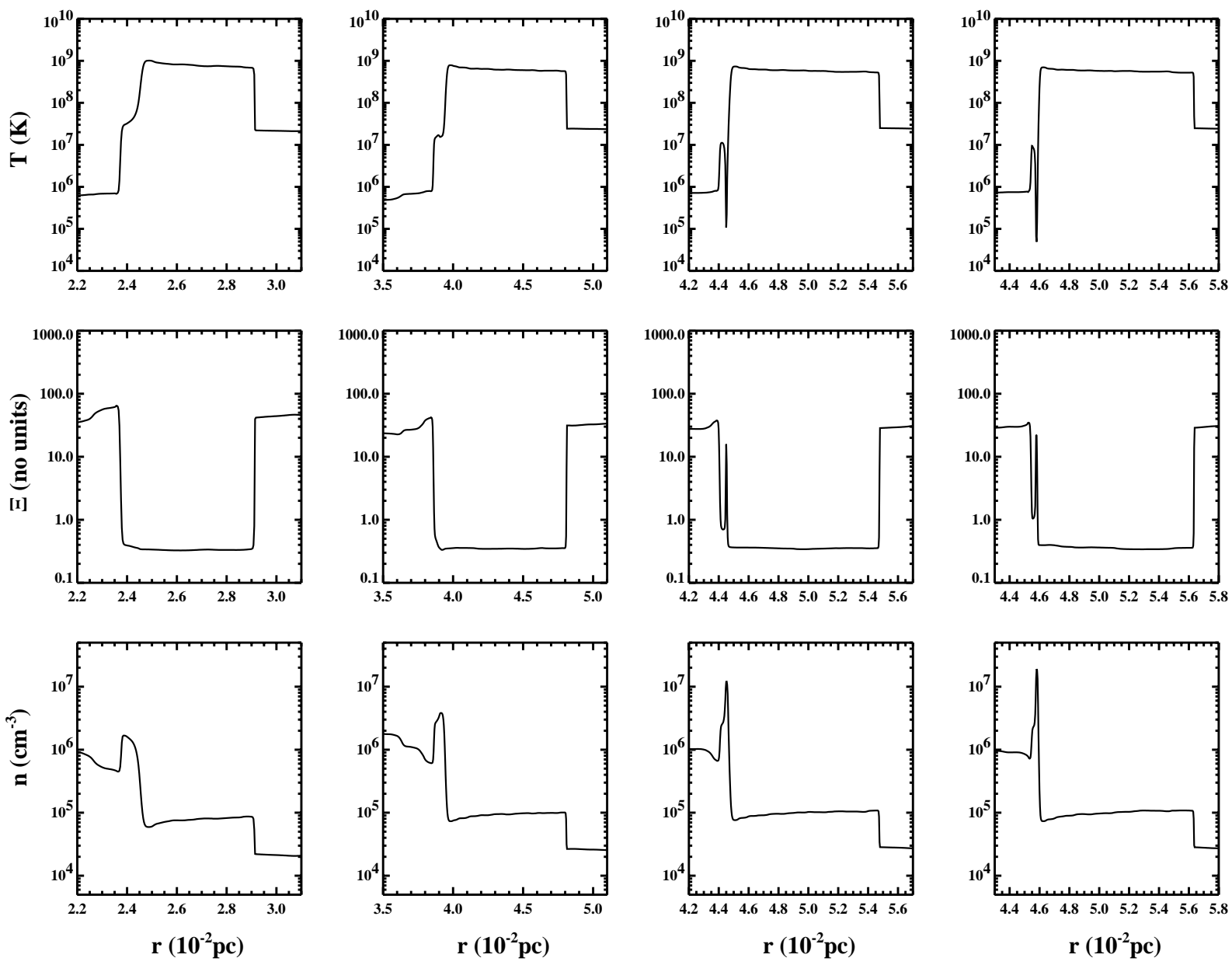

Fig. 7. As Fig. 6 (Model B) but in this simulation (Model C) the ambient medium has a flow velocity towards the remnant of $3000 \mathrm{~km} \mathrm{~s}^{-1}$. From left to right the corresponding times are $5 \mathrm{yr}, 10 \mathrm{yr}, 12 \mathrm{yr}$ and $12.5 \mathrm{yr}$. In contrast to the results for a stationary medium, cool clouds can form in this case. Note also the more rapid evolution of the remnant with respect to the case shown in Fig. 6.

although a high ambient density does not inhibit the formation of a cool region, at some level it results in such rapid evolution that these regions are destroyed without making any substantial contribution to the HIL emission.

The distance to the central engine influences the value of $\Xi_{\text {shk }}$ through the ionzing flux (Eq. (A.8)) Therefore there is an upper limit to $\Xi_{\mathrm{w}}$ after which not even SNe in the densest environments will be able to produce cool regions. If the ambient medium is in motion relative to the remnant, the parameter space over which cool regions can form is extended (Model B (Fig. 6) vs. Model C (Fig. 7)).

The second constraint is that the ambient medium needs to be dense enough for the cooling timescale of the shocked gas to be less than the age of the remnant at the time when the reverse shock reaches ejecta which do not have a strongly radially dependent density. If this is not the case no cool regions can form, as illustrated by Model B (Fig. 6).

\section{Discussion}

The results in Sect. 3 demonstrate that it is possible to cool shocked supernova ejecta down to $T \sim 10^{4} \mathrm{~K}$ in the inner regions of a QSO. Although our results differ from the original proposals of Dyson \& Perry (1982) and Perry \& Dyson (1985), which were for the shocked ambient medium to cool, the resulting cool gas nevertheless has properties (densities, column depths, velocities and ionization parameters) compatible with those inferred for gas emitting the high ionization lines in QSOs. We have additionally shown from three separate calculations that this model is able to cover the parameter range associated with observations of BELRs, and have highlighted scenarios which are outside of this range and preclude the formation of cool regions.

We now compare our results to some of the observational correlations that have been determined. Korista et al. (1995) noted that the blue and red wings respond faster to continuum changes than the core. If one assumes that the expansion velocity of the supernova remnant is 
the dominant velocity component, this observation can be explained with our model in the following way. Remnants which are surrounded by the highest ambient densities evolve most rapidly, and therefore form cool regions with the highest expansion velocities. If the ambient density increases towards the central engine, there will be a tendency to form cool regions with, on average, higher velocities. Being close to the central engine, these regions respond more rapidly to any continuum changes, as the observed correlation requires. Even if $v_{\text {exp }}$ is not the dominant component, the above argument implies that our assumed model nevertheless provides a statistical bias in this direction.

There now appears to be conclusive evidence that the higher ionization lines respond faster than lower ionization lines to changes in the continuum variability (see references in Fromerth \& Melia 2001). If the LIL are formed in an accretion disc close to the central engine, this implies that the thermal timescale in the disc is longer than the corresponding timescale for the HIL. Alternatively the delay could be explained if the bulk of the LIL heating is due to back-scattered X-rays from the gas responsible for the HILs and the general ambient medium (see Collin-Souffrin et al. 1988). Therefore, our model is consistent with these observations.

Our calculations show that it is the shocked ejecta which cool to form the HILs, and that once the ejecta core reaches the reverse shock, the shocked region rapidly depressurizes. Since this presumably leads to the destruction of any cool regions, it seems sensible to suppose that for the remnant parameters we have chosen, an upper limit to the HIL mass would be $2.5 M_{\odot}$ per remnant. With further appropriate assumptions we can then estimate the required supernova rate needed to sustain the mass in the gas responsible for the HIL. With the assumption that each remnant can eventually cool $\sim 2.5 M_{\odot}$ of gas, and that the lifetime of the cool gas in the remnant is of order 10 yr, to sustain $\sim 25 M_{\odot}$ of gas in the HIL would require a supernova rate of $\sim 2 \mathrm{yr}^{-1}$. Although our estimates are very uncertain, this rate is acceptable: certainly for high luminosity QSOs, supernova rates $\sim 10 \mathrm{yr}^{-1}$ are conceivable (Terlevich et al. 1992). A less steep density distribution of the ejecta envelope (if $n<12$ more mass would be available per SN), and the suppression of dust formation in intermediate-mass AGB stars in the BELR region (which may reduce the minimum zero-age main sequence mass required for supernovae; Hartquist et al.1998) are two additional possibilities which could further reduce our estimate of the required supernova rate. Additionally, it is possible that conditions exist for the swept-up ambient material to also cool to temperatures appropriate for the HIL, as the cooling time estimated by Eq. (A.7) is temperature independent. The fact that the reverse shocked material cools first in our simulations is simply due to enhanced cooling by line emission starting earlier due to its lower initial temperature. In Model A where $t_{\text {cool }}<t_{\text {dyn }}$ we therefore might expect the swept-up ambient material to form HIL gas as well. At $t=t_{\mathrm{dyn}}$, the forward shock is estimated to be at a radius of $0.03 \mathrm{pc}$ and to have swept up 1.6 $M_{\odot}$ of ambient gas. This gas could therefore be a significant contributer to the total HIL emission.

In this first paper we have been solely interested in the question of whether cool regions could form from supernova shocks given that they are bathed in the hard radiation flux of the central engine. For simplicity we restricted our modelling to the simplest 1D approach, and assumed solar metallicites in our calculations. The fact that the ejecta (and also the swept up medium) may be responsible for the HIL emission warrants a careful consideration in future models. We will also perform calculations on $2 \mathrm{D}$ axisymmetric hydrodynamic grids.

Another future goal is the inclusion of the effect of the QSO radiation field on the dynamics of the remnant. At high temperatures, the effective cross-section of gas is the Thomson scattering cross-section, $\sigma_{\mathrm{T}}$. Once the gas begins to cool, the effective cross-section, $\bar{\sigma}$, increases, enhancing the radiative driving. As noted by Williams et al. (1999), $\bar{\sigma} / \sigma_{\mathrm{T}}$ increases to roughly 2 at $10^{6} \mathrm{~K}, 40$ at $10^{5} \mathrm{~K}$, and to $\geq 10^{4}$ when the gas has fully cooled (see also Arav \& Li 1994; Arav et al. 1994). For gas cooler than $\sim 5 \times 10^{4} \mathrm{~K}$, it is reasonable to take the radiative acceleration as $g=$ $7 \times 10^{14} \rho$ (Röser 1979; Dyson et al. 1981). We expect that substantial radiative driving will critically alter the dynamics of the interaction between the supernova ejecta and the ambient medium (e.g. Falle et al.1981; Williams 2000).

Although we have chosen to model the interaction of supernova ejecta with the ambient medium, it is possible that a wind from a group of early-type stars may also provide the necessary conditions for the formation of cool regions. This interaction may be more relevant in the nuclei of Seyfert galaxies, since supernova explosions will evacuate all but the most tightly bound gas in them (Perry \& Dyson 1985). Finally it is clear from our models that whilst the supernova-QSO wind interaction is conceptually simple, the BELR is likely to be a very complicated region in practice.

Acknowledgements. JMP would like to thank PPARC for the funding of a PDRA position. We would like to thank R. J. R. Williams and R. Coker for many helpful conversations and T. Woods for the use of his cooling and heating tables. This research has made use of Nasa's Astrophysics Data System Abstract Service. We would also like to thank an anonymous referee whose suggestions improved this paper.

\section{Appendix A: Derivation of $t_{\mathrm{dyn}}$ and $\Xi_{\text {shk }}$}

From Chevalier (1982), the radius of the contact discontinuity for a supernova remnant expanding into a stationary, constant density ambient medium $\left(\rho_{\mathrm{w}}=n_{\mathrm{w}} \mu_{\mathrm{w}} m_{\mathrm{H}}\right.$, where $\mu_{\mathrm{w}}$ is the average mass per particle), is

$R_{\mathrm{c}}=\left(\frac{A g^{n}}{n_{\mathrm{w}} \mu_{\mathrm{w}} m_{\mathrm{H}}}\right)^{1 / n} t^{(n-3) / n}$ 
where $A$ is a constant determined by the steepness of the ejecta profile $(n)$. The radius of the reverse shock is

$R_{2}=\frac{R_{2}}{R_{\mathrm{c}}} R_{\mathrm{c}}$

where $R_{2} / R_{\mathrm{c}}$ is also a function of $n$. For $n=12$, Chevalier's Table 1 gives $A=0.19$ and $R_{2} / R_{\mathrm{c}}=0.974$. The velocity of the pre-shock ejecta at the reverse shock is

$\left.v_{\mathrm{ej}}\right)_{R_{2}}=R_{2} / t$.

The dynamical time (i.e. the time at which the ejecta core reaches the position of the reverse shock) can be obtained from the ratio

$$
\frac{t_{\mathrm{dyn}}}{t}=\left(\frac{v_{\mathrm{c}}}{\left.v_{\mathrm{ej}}\right)_{R_{2}}}\right)^{-n / 3},
$$

where $\left.v_{\text {ej }}\right)_{R_{2}}$ is the velocity of the pre-shock ejecta at the reverse shock at time $t$. Substituting Eqs. (1), (3) and (A.3) we obtain

$$
\begin{aligned}
t_{\mathrm{dyn}}= & \left(\frac{A}{4 \pi(n-\delta) n_{\mathrm{w}} \mu_{\mathrm{w}} m_{\mathrm{H}}}\right)^{1 / 3}\left(\frac{R_{2}}{R_{\mathrm{c}}}\right)^{n / 3} \\
& \times \frac{[2(5-\delta)(n-5) E]^{-1 / 2}}{\left[(3-\delta)(n-3) M_{\mathrm{ej}}\right]^{-5 / 6}}
\end{aligned}
$$

Since the shocked gas is initially at very high temperatures, we make the assumption that the dominant cooling during this interval is inverse Compton. The radiative losses $\left(\mathrm{erg} \mathrm{cm}^{-3} \mathrm{~s}^{-1}\right)$ are given by

$\dot{E} \approx 2.2 \times 10^{7} \frac{L_{47}}{r_{\mathrm{pc}}^{2}} T_{8} \rho$

(Perry \& Dyson 1985). The cooling timescale is independent of density and temperature, and is given by

$t_{\text {cool }}=\frac{E}{\dot{E}} \approx 18 \frac{r_{\mathrm{pc}}^{2}}{\mu_{\mathrm{s}} L_{47}} \mathrm{yrs}$,

where $\mu_{\mathrm{s}}$ is the average mass per particle for the shocked material. For the post-shock gas to cool down to approximately $T=2 \times 10^{4} \mathrm{~K}$, we require $t_{\text {cool }} \lesssim t_{\text {dyn }}$.

We now derive an expression for the evolution of the ionization parameter of post-shock gas cooled back to equilibrium with the radiation field, $\Xi_{\text {shk }}$. From Perry \& Dyson (1985) we have

$\Xi_{\mathrm{shk}}=\frac{L_{\mathrm{ion}}}{4 \pi r^{2} n_{\mathrm{s}} k T_{\mathrm{s}} c}=2.0 \times 10^{14} \frac{L_{\mathrm{ion}}, 47}{r_{\mathrm{pc}}^{2} n_{\mathrm{s}} T_{\mathrm{s}}}$,

where $n_{\mathrm{s}}$ is the number density of the shocked material, $T_{\mathrm{s}}$ is the immediate post-shock temperature of the material and is given by

$T_{\mathrm{s}}=\frac{3}{16} \frac{\mu_{\mathrm{s}} m_{\mathrm{H}}}{k} v_{\mathrm{r}}^{2}$,

and $v_{\mathrm{r}}$ is the velocity of the pre-shock ejecta in the frame of the reverse shock. The velocity of the reverse shock is

$v_{R_{2}}=\frac{R_{2}}{R_{\mathrm{c}}} v_{\mathrm{cd}}=\frac{n-3}{n} \frac{R_{2}}{R_{\mathrm{c}}}\left(\frac{A g^{n}}{n_{\mathrm{w}} \mu_{\mathrm{w}} m_{\mathrm{H}}}\right)^{1 / n} t^{-3 / n}$, so using Eq. (A.3) we obtain

$$
\begin{aligned}
v_{\mathrm{r}}= & {\left[1-\left(\frac{n-3}{n}\right)\right] \frac{R_{2}}{R_{\mathrm{c}}}\left(\frac{A}{4 \pi n_{\mathrm{w}} \mu_{\mathrm{w}} m_{\mathrm{H}}(n-\delta)}\right)^{1 / n} } \\
& \times \frac{[2(5-\delta)(n-5) E]^{(n-3) / 2 n}}{\left[(3-\delta)(n-3) M_{\mathrm{ej}}\right]^{(n-5) / 2 n}} t^{-3 / n}
\end{aligned}
$$

which at $t=t_{\mathrm{dyn}}$ simplifies to

$\left.v_{\mathrm{r}}\right)_{t_{\mathrm{dyn}}}=\left[1-\left(\frac{n-3}{n}\right)\right] v_{\mathrm{c}}$.

Combining Eqs. (A.8), (A.9) and (A.12), and noting that as it is the reverse shock which cools, $n_{\mathrm{s}}=4 n_{\mathrm{w}} \rho_{2} / \rho_{1}=$ $4 \rho_{\mathrm{w}} \rho_{2} /\left(\rho_{1} \mu_{\mathrm{w}} m_{\mathrm{H}}\right)$, we obtain

$\left.\Xi_{\mathrm{shk}}\right)_{t_{\mathrm{dyn}}} \approx \frac{1}{27} \frac{\rho_{1}}{\rho_{2}} \frac{L_{\mathrm{ion}}, 47}{r_{\mathrm{pc}}^{2} n_{\mathrm{w}} \mu_{\mathrm{w}} m_{\mathrm{H}}}\left(\frac{1}{\left[1-\left(\frac{n-3}{n}\right)\right] v_{\mathrm{c}}}\right)^{2}$

Values for $\rho_{1} / \rho_{2}$ are given in Chevalier (1982).

\section{References}

Alexander, T., \& Netzer, H. 1994, MNRAS, 270, 781

Alexander, T., \& Netzer, H. 1997, MNRAS, 284, 967

Arav, N., \& Li, Z.-Y. 1994, ApJ, 427, 700

Arav, N., Li, Z.-Y., \& Begelman, M. C. 1994, ApJ, 432, 62

Artymowicz, P., Lin, D. N. C., \& Wampler, E. J. 1993, ApJ, 409, 592

Band, D. L., \& Liang, E. P. 1988, ApJ, 334, 266

Beltrametti, M. 1981, ApJ, 250, 18

Blondin, J. M., Borkowski, K. J., \& Reynolds, S. P. 2000 [astro-ph 0010285]

Carroll, T. J. 1985, MNRAS, 214, 321

Carroll, T. J., \& Kwan, J. 1985, ApJ, 288, 73

Cassidy, I., \& Raine, D. J. 1996, A\&A, 310, 49

Chevalier, R. A. 1976, ApJ, 207, 872

Chevalier, R. A. 1982, ApJ, 258, 790

Chevalier, R. A., \& Fransson, C. 1994, ApJ, 420, 268

Clavel, et al. 1991, ApJ, 366, 64

Collin-Souffrin, S., Dumont, S., Joly, M., \& Pequignot, D. 1986, A\&A, 166, 27

Collin-Souffrin, S., Dumont, S., \& Tully, J. 1982, A\&A, 106, 362

Collin-Souffrin, S., Dyson, J. E., McDowell, J. C., \& Perry, J. J. 1988, MNRAS, 232, 539

Dyson, J. E., Falle, S. A. E. G., \& Perry, J. J. 1981, MNRAS, 191,397

Dyson, J. E., \& Perry, J. J. 1982, In Third European IUE Conference

Emmering, R. T., Blandford, R. D., \& Shlosman, I. 1992, ApJ, 385,460

Falle, S. A. E. G., \& Komissarov, S. S. 1996, MNRAS, 278, 586

Falle, S. A. E. G., \& Komissarov, S. S. 1998, MNRAS, 297, 265

Falle, S. A. E. G., Perry, J. J., \& Dyson, J. E. 1981, MNRAS, 195, 397

Field, G. B. 1965, ApJ, 142, 431

Fromerth, M. J., \& Melia, F. 2001, ApJ, 549, 205

Gaskell, C. M. 1988, ApJ, 325, 114

Gaskell, C. M., Brandt, W. N., Dietrich, M., Dultzin-Hacyan, D., \& Eracleous, M. 1999, Structure and Kinematics of Quasar Broad Line Regions, ASP Conf. Ser., 175 
Hamann, F., \& Ferland, G. 1992, ApJ, 391, L53

Hartquist, T. W., Bertoldi, F., Durisen, R. H., et al. 1998, The Molecular Astrophysics of Stars (Oxford University Press) Jones, E. M., Smith, B. W., \& Straka, W. C. 1981, ApJ, 249, 185

Kazanas, D. 1989, ApJ, 347, 74

Korista, K. T., Alloin, D., Barr, P., et al. 1995, ApJS, 97, 285

Krolik, J. H., McKee, C. F., \& Tarter, C. B. 1981, ApJ, 249, 422

Kwan, J., \& Carroll, T. J. 1982, ApJ, 261, 25

Kwan, J., \& Krolik, J. H. 1981, ApJ, 250, 478

Laor, A., \& Draine, B. T. 1993, ApJ, 402, 441

Marcaide, J. M., Alberdi, A., Ros, E., et al. 1997, ApJ, 486, L31

Nadyozhin, D. K. 1985, ApSS, 112, 225

Osterbrock, D. E. 1993, RMAA, 26, 65

Osterbrock, D. E., \& Matthews, W. G. 1986, ARA\&A, 24, 171

Parker, E. N. 1963, Interplanetary Dynamical Processes (New York, Interscience)

Perry, J. J., \& Dyson, J. E. 1985, MNRAS, 213, 665

Roos, N. 1992, ApJ, 385, 108
Röser, H.-J. 1979, A\&A, 80, 179

Scoville, N., \& Norman, C. 1988, ApJ, 332, 163

Shlosman, I., Begelman, M. C., \& Frank, J. 1990, Nature, 345, 679

Shlosman, I., Vitello, P. A., \& Shaviv, G. 1985, ApJ, 294, 96

Smith, M. D., \& Raine, D. J. 1985, MNRAS, 212, 425

Sulentic, J. W., Marzani, P., Dultzin-Hacyan, D., Calvani, M., \& Moles, M. 1995, ApJ, 445, L85

Suzuki, T., \& Nomoto, K. 1995, ApJ, 455, 658

Terlevich, R., Tenorio-Tagle, G., Franco, J., \& Melnick, J. 1992, MNRAS, 255, 713

Truelove, J. K., \& McKee, C. F. 1999, ApJS, 120, 299

Williams, R. J. R. 2000, MNRAS, 316, 803

Williams, R. J. R., et al. 1999, MNRAS, 310, 913

Williams, R. J. R., \& Perry, J. J. 1994, MNRAS, 269, 538

Wills, B. J., Netzer, H., \& Wills, D. 1985, ApJ, 288, 94

Woods, D. T., Klein, R. I., Castor, J. I., McKee, C. F., \& Bell, J. B. 1996, ApJ, 461, 767

Woosley, S. E., Pinto, P. A., \& Ensman, L. 1988, ApJ, 324, 466

Zurek, W. H., Siemiginowska, A., \& Colgate, S. A. 1994, ApJ, 434, 46 